\title{
Yoga y formación del profesorado
}

\section{Yoga and teacher training}

\author{
Lourdes Fuente Torre ${ }^{1}$ (D) ; Agustín de la Herrán Gascón ${ }^{2}$ \\ ${ }^{1}$ CEIP Miguel de Unamuno (Madrid), España; ${ }^{2}$ Universidad Autónoma de Madrid (UAM)
}

\begin{abstract}
Resumen
El yoga es una de las metodologías formativas, basadas en la meditación, más antiguas y contrastadas. Originario de la India, en los últimos años ha incrementado su presencia en la educación occidental. Cada vez hay más centros educativos en los que se desarrolla yoga para niños. Sin embargo, su relación con la formación del profesorado sigue siendo un reto pedagógico apenas atendido. El objetivo del estudio es conocer cómo influye la práctica sistemática del yoga en la conciencia del docente de educación infantil y primaria. Se trata de comprender si esta práctica es relevante desde la perspectiva de la experiencia formativa y profesional de los docentes. Se parte de una conceptualización de conciencia, tanto desde un punto de vista "etic", o de la teoría, como "emic", o de los participantes, según la terminología de Harris (1980). Para responder al objetivo, se llevan a cabo veintiún estudios de caso, mediante entrevistas en profundidad. Los participantes son dieciséis maestros de educación infantil y primaria que practican yoga sistemáticamente, y cinco maestros de yoga, expertos y reconocidos. Los resultados, obtenidos mediante análisis de contenido, indican que la práctica del yoga ayuda a desarrollar la conciencia del docente de un modo efectivo, y otras cualidades importantes para la función docente, estrechamente vinculadas a la conciencia, como la empatía y la humildad didáctica. Se concluye que la práctica del yoga es una metodología formativa que contribuye al desarrollo personal y profesional de los docentes.
\end{abstract}

Palabras clave: yoga; formación; conciencia; desarrollo personal y profesional del docente; educación.

\begin{abstract}
Yoga is one of the oldest and most contrasted meditation-based training methodologies. Originally from India, in recent years has increased its presence in Western education. There are more and more educational centers where yoga is developed for children. However, its relationship with teacher training remains a barely attended pedagogical challenge. The objective of the study is to know how the systematic practice of yoga influences the consciousness of the teacher of early childhood and primary education. It is about understanding, if this practice is relevant from the perspective of the formative and professional experience of the teachers. It starts from a conceptualization of consciousness, both from an "ethic" point of view, or from the theory, as "emic", or from the participants, according to the terminology of Harris (1980). To answer the objective, twenty-one case studies are conducted through in-depth interviews. The participants are sixteen teachers of early childhood and primary education who practice yoga systematically, and five yoga teachers, experts and recognized. The results, obtained through content analysis, indicate that the practice of yoga helps to develop the teacher's consciousness in an effective way, and other important qualities for the teaching function closely linked to consciousness, such as empathy and didactic humility. It is concluded that the practice of yoga is a formative methodology that contributes to the personal and professional development of the teachers.
\end{abstract}

Keywords: yoga; training; awareness; personal and professional development of the teacher; education

Como citar (APA):

Herrán, A. de la \& Fuente, L. (2021). Yoga y formación del profesorado. Revista Iberoamericana de Educación, 85(2),73-94. https://doi.org/10.35362/rie8523936 


\section{Introducción}

En la investigación pedagógica hay un alto interés y mucha preocupación científica por el desarrollo personal y profesional del docente y por la mejora de su práctica. Hay unanimidad en la relevancia de la reflexión, entendida como ámbito clave para alcanzar ese objetivo. Autores reconocidos, como Korthagen (2010), Pérez (2010), Torre (2010), Gimeno (1998) o Day (2006) propugnan la atención a la reflexión del docente como ámbito formativo y medio para lograr el desarrollo humano profesional y el cambio didáctico.

Sin embargo, desde el enfoque radical e inclusivo de la formación, se ha observado que la reflexión sobre la práctica sólo cambia superficialmente, cuando lo hace, y no trae, necesariamente, cambios profundos en la enseñanza, ni en el desarrollo profesional y personal de los docentes (Herrán, 2011a, 2014, 2018; Ramírez y Herrán, 2012). Por esta razón, se deduce que el enfoque técnico reflexivo, pudiendo ser necesario en muchas situaciones, no es suficiente, profesional o epistemológicamente, si la referencia es una educación más cabal.

La observación fundamental es que tanto la práctica como la reflexión son efectos de quien las realiza. Por tanto, si la formación del profesorado se centra en los efectos, será, bien superficial, bien profundizable, completable con una atención al interior. ¿Cabe, entonces, un enfoque más profundo centrado, además de en la reflexión y en la práctica, en quien realiza la práctica? El enfoque radical e inclusivo de la formación incluye como prioridad formativa de cualquier educador el acceso directo al autoconocimiento a través de la meditación (Herran, 1993, 1995, 1996, 1997, 1998, 2003, 2004, 2011a, 2014, 2016a, 2016b, 2017a, 2017b, 2017c, 2017d, 2017e, 2018). La meditación es una metodología y el autoconocimiento un ámbito altamente complementario a la reflexión sobre la práctica, pero no tiene nada que ver con la acción del pensamiento o la indagación. Por tanto, un enfoque complejo que atendiese ambas vertientes sería, a priori, más completo que cualquiera de los dos considerados aisladamente. En la medida en que hoy la perspectiva técnico reflexiva define la normalidad en la formación del profesorado y de la Didáctica, la inclusión de la meditación pudiera ser oportuna y eficaz, si de formación personal y profesional más plenas se trata. 
La reflexión no llega a toda la práctica ni a toda la persona del docente. No es que el pozo sea demasiado profundo; es que la cuerda es demasiado corta. Por ejemplo, no llega al autoconocimiento y casi nunca llega al cambio de la mala práctica generada por egocentrismo docente (Herrán y González, 2002). Entre otras razones, asociables a nuestro sistema educativo, esto ocurre por el enfoque normal de la formación del profesorado, que excluye de los ámbitos básicos el autoconocimiento, el egocentrismo docente y la conciencia. No obstante, los tres retos o ámbitos son accesibles o comprensibles desde el tercero, la conciencia8).

Si la tendencia del docente es egocéntrica y la conciencia está inhibida o no desarrollada -entre otras cosas, porque no se incluye en la formación normal o no se habla de ella-, nos encontraremos con la imposibilidad de desarrollar la conciencia en el niño. Es más, será difícilmente perceptible la conciencia de la falta de conciencia y la consecuente necesidad autoeducativa de desarrollarla en sí y con el otro. Por tanto, un punto de partida para mejorar la educación de todos puede ser atender al desarrollo de la conciencia del docente, no sólo para mejorar la educación, sino, yendo más allá en los planteamientos y objetivos cercanos, para contribuir a la mejora de la sociedad y de la humanidad.

Desde la Edad Antigua, tanto en el Oriente clásico como en Occidente, se ha valorado el autoconocimiento. En el siglo -VI, dos maestros influyen decisivamente en el desarrollo del conocimiento de Occidente y de Oriente: Sócrates (Platón, 1969), semilla de la Filosofía y de la Pedagogía, habla de autoconocimiento, pero no explica cómo acceder o cultivarse en su sentido. Siddhartha Gautama (Buda, 1977), en cambio, realiza una aportación metodológica y experimental en torno a la meditación. Antes que él, el yoga se desarrollaba en la India como práctica meditativa orientada tanto a la salud física y psíquica, como a la propia educación y formación. Específicamente, tanto la meditación como el yoga se orientaban al desarrollo de la conciencia y al conocimiento de sí mismo.

En esta investigación se plantea comprobar si una vía de conciencia clásica, ancestral y contrastada experimentalmente durante milenios como es el yoga, incide o afecta a la conciencia del docente, y si el desarrollo de esta conciencia, en su caso, puede ayudar concreta y eficazmente al desarrollo 
personal y profesional docente y a la mejora de su práctica didáctica. Si se verificase, significaría que el yoga es una metodología importante, realizable y conveniente en la formación de los profesores.

Que la práctica del yoga ayuda a la salud física y al bienestar personal de quien lo practica es algo verificado en numerosas investigaciones relevantes (Correa y Guerra, 2014; Jyoti, Rani y Gandotra, 2015). También se han confirmado beneficios significativos de salud y bienestar en docentes (Deniz, Torres y Alcantar, 2010). La presente investigación se apoya es éstas, pero se intenta centrar en la relación del yoga con el desarrollo de la conciencia y la formación del docente. Como se ha apuntado, la investigación sobre formación con base en la conciencia de los profesores es muy escasa.

\section{Marco Teórico}

El neurocientífico Damasio (2010) conceptualiza la conciencia como "la capacidad de darse cuenta de la realidad". Análogamente la define Calle (2017), maestro de yoga y gran conocedor de la tradición meditativa de la India, al expresar que "la conciencia es la capacidad de darse cuenta, de percibir y percibirse, de conectarse con el aquí-ahora a través de la poderosa energía de la atención". Herrán (1998) propone un sistema para ordenar clasificaciones de estados de conciencia, reuniéndolos en siete grupos. Por su parte, Goleman y Senge (2016) clasifican la conciencia en tres ámbitos de aplicación, diferenciando entre autoconciencia o conciencia de sí, conciencia del otro y conciencia de sistemas más amplios, diferenciando subámbitos (Figura 1):

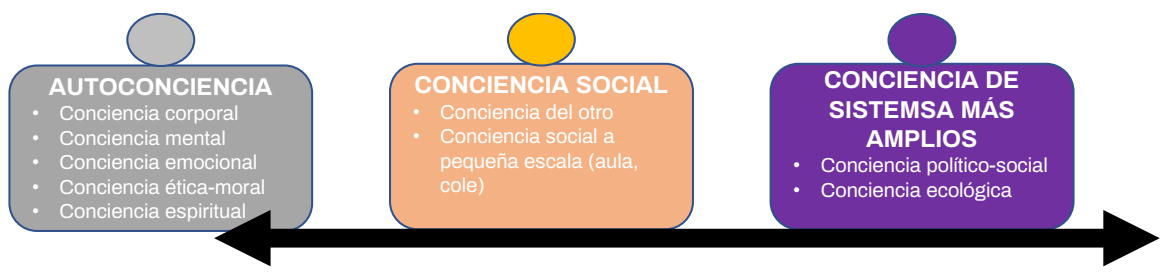

Figura 1. Ámbitos de aplicación de la conciencia. Fuente: Goleman y Senge (2016). 
Nuestra conceptuación de conciencia, aplicada a la educación y al yoga como metodología formativa, incluye una perspectiva holística. No se ciñe al pensamiento, ni equivale a metacognición. Se refiere a la totalidad de la persona, en todos los planos constitutivos y de desarrollo: físico, existencial, emocional, mental y espiritual, y a su mutua interrelación. Globalmente, se relaciona con la conciencia de la totalidad del mundo y con nuestra relación vital con él, para la evolución personal, social y de la humanidad. La conciencia es desarrollable, según muestran los psicólogos transpersonales, y su desarrollo pleno atravesaría por varias etapas. Para González-Zarza (2006) los niveles de desarrollo de la conciencia son: preegoico, egoico, prepersonal, personal, organísmico-social y transpersonal. Se corresponderían con las etapas de Piaget completadas con las teorías psicoanalíticas de Freud y Jung. Por su parte, Wilber (2007) distingue varios niveles de la conciencia humana: nivel de persona, nivel de ego, organismo total y nivel unidad.

Desde las disciplinas meditativas y formativas, se habla de "vías de conciencia" (Herrán, 1995, 1998) o de caminos para el desarrollo de la conciencia. Para Kornfield (2003), las diferentes técnicas de desarrollo de la conciencia modifican la forma en la que nos relacionamos con nuestras experiencias. Además, tratan de promover cualidades internas, como la concentración, la serenidad, la profundización y el equilibrio de nuestra conciencia. Dentro de éstas, el yoga es una técnica o vía para el trabajo interior, con el fin último del desarrollo o evolución consciente. "La palabra ‘yoga' deriva de la raíz sánscrita yug, término polisémico "que significa ligar, unir, sujetar, uncir, concentrar la atención, utilizar y aplicar" (Iyengar, 2007, p. 201). Por medio del trabajo interior, a través del yoga, se llega al autoconocimiento en diversos niveles de profundidad, pasando por la autoconciencia, que hace que nos demos cuenta de nuestras fortalezas, debilidades, estados mentales y emocionales, condicionamientos, identificaciones, etc. El enfoque holístico de la educación busca enfatizar en el ser, más que en el hacer y en el tener. Como propugna Mayor Zaragoza, la educación no debe basarse sólo en el saber o tener, sino también en el ser: el ser de nuestros alumnos, lo más valioso de su vida y lo más importante para un verdadero desarrollo humano, cuestión nada baladí ante la actual situación tanto social como medioambiental.

Desde la perspectiva del enfoque radical e inclusivo, tanto en el plano personal como social, la educación es un proceso que transcurre del egocentrismo a la conciencia. Esta acepción requiere la inclusión de estos constructos, 
junto al autoconocimiento, en la formación y en la ciencia normal. Tanto el ego como la conciencia son constructos y fenómenos básicos para la comprensión del enfoque meditativo y autoformativo de la tradición yóguica y de Siddhartha Gautama, a la que ya se hizo referencia. Con esta mirada pedagógica, la educación habitual, a la que se refieren los organismos internacionales, los sistemas educativos y los currícula, se ocupa eminentemente de lo exterior del ser humano. Se entiende por exterior lo interesante para la existencia, a saber, lo social o profesionalmente necesario, que tiende a enfatizar el tener y el hacer sobre el ser (Fromm, 1991). Este enfoque, sin embargo, no es dual, sino dialéctico y sintético, y lo que propone es que la educación tiene que ocuparse de todo a la vez: de lo exterior y de lo interior, de lo demandado y de lo importante no demandado, de retos coyunturales y perennes, del aprendizaje y del descondicionamiento, de competencias y de la conciencia, de la reflexión y la meditación, etc. De ahí su calificativo de 'inclusivo'.

Desde una perspectiva global, holística y transpersonal, la educación es un recurso clave de nuestro tiempo y el recurso cultural que puede fomentar el proceso de desarrollo personal y social durante toda la vida (Sanches, 2008). Desde el enfoque radical e inclusivo (Herrán, 2014), nuestra educación no es plena, sino incompleta, tiene carencias graves, porque desatiende lo interior, al no incluir el egocentrismo, la conciencia, el autoconocimiento, la muerte, la humanidad, etc.) como de la metodología autoformativa. Un modo de avanzar en su proceso de compleción es incluyendo la interiorización y el desarrollo de la conciencia a través de la meditación. Dentro de las disciplinas meditativas, una de las más contrastadas y antiguas es el yoga. Este estudio se apoya en la teoría transpersonal, holística y con el enfoque radical e inclusivo de la educación, y propone como fin último de la educación el desarrollo o creciente despertar de la conciencia del alumno a través del yoga aplicado a la formación de los profesores.

Así pues, el sentido último de la educación, en la que la práctica del yoga adquiere todo su sentido, pasa por mirar más la evolución en complejidad de conciencia de la persona, de la sociedad y de la humanidad, que su interés, rentabilidad o eficacia personal o social. La orientación del proceso formativo a través de la persona toma como referencia el autoconocimiento, al que cabe aproximarse a través del despertar de la conciencia y, desde él, a una experiencia de vida más centrada y con sentido. La metodología didáctica para una enseñanza con base en la conciencia se apoya en un trabajo per- 
sonal previo o coherente -en este contexto, desde la práctica sistemática del yoga y durante un tiempo suficiente- de profesores o educadores. Es decir, la pauta es que los educadores y docentes primero se ocupen de su formación y de su práctica, antes de enseñar nada a los demás, como apuntaba Confucio (1969).

Reconociendo la amplitud y profundidad del campo en que nos situamos, se pretende conocer cómo influye la práctica sistemática del yoga en la conciencia del docente de educación infantil y primaria. Para ello, nos interesa conocer, desde una perspectiva fenomenológica, cómo conceptúan la conciencia y cómo interpretan su experiencia formativa los profesores practicantes de yoga que participan en el estudio. Será interesante contrastar sus testimonios con las definiciones de expertos. A partir de ahí, se podrá deducir si hay influencia, desde una la perspectiva "emic" o teórica, entre la realización de una práctica como el yoga y el desarrollo de la conciencia del docente.

Los objetivos específicos de investigación, que concretan el objetivo general, son:

1. Describir lo que los docentes que participan en el estudio entienden por conciencia.

2. Comprender y explicitar la relación que se establece entre la conciencia y la educación, desde su perspectiva experimental.

3. Analizar, comprender y explicar las implicaciones que la práctica sistemática del yoga tiene en la conciencia del docente.

4. Verificar y, en su caso, clarificar si una práctica como el yoga puede ser beneficiosa para el desarrollo personal y profesional docente.

\section{Metodología de la investigación}

El enfoque del estudio es cualitativo. La investigación cualitativa parte de la tradición filosófica hermenéutica de mediados del siglo XX. Considera que la ciencia que investiga los fenómenos humanos debe ser interpretativa y buscar la comprensión en profundidad de los procesos de estudio y de las relaciones, sin huir de la subjetividad. En este sentido, González (2008) afirma que: "La subjetividad es el fenómeno; la objetividad es una pretensión". Para Martínez (1988) el epicentro de una investigación cualitativa 
e interactiva son los valores y la subjetividad de los participantes. En este sentido, el enfoque comprensivo que proviene de la investigación en ciencias humanas recupera al actor y sus intenciones:

El enfoque comprensivo que deviene de la tradición de las ciencias humanas se caracteriza por reconocer la trascendencia de la subjetividad humana y del contexto histórico en la confrontación de la vida social, recuperando al actor como elemento central que realiza acciones con sentido, es decir con intención (Aguayo, 2013, p. 37).

Dentro del enfoque cualitativo, la investigación se incluye en una perspectiva fenomenológica o subjetivista, que "da prioridad a la perspectiva del sujeto, de la persona, en la interpretación de la realidad" (Tójar, 2006, p. 66). Desde nuestra perspectiva e intencionalidad investigadora reivindicamos, además, la asociación del conocimiento con el cuerpo y los procesos derivados de él: sensaciones, percepciones, intuición, deseos, sentimientos, intereses, etc., para recuperar el significado holístico asociado a la experiencia, tanto propia como del otro. Es más, al tratarse de educación y estar muy presente la relación formativa con y sobre personas, se pone en juego la 'experiencia del tú', del encuentro con el otro (Buber, 1977), la transformación personal que eso supone y lo que, desde ahí, pueda decirse del saber adquirido o desprendido, desde la conciencia actualizada.

El diseño de investigación es el estudio de casos, definido por Yin (2009) como un método que permite un estudio holístico y significativo de un acontecimiento o fenómeno contemporáneo, dentro del contexto real en que se produce. Se seleccionaron veintiún participantes, con el siguiente perfil: maestros y maestras de educación infantil o primaria, practicantes sistemáticos de yoga durante al menos dos años y durante al menos cinco de los siete días de la semana, al menos 20 minutos cada meditación. Se intentó que fueran tanto hombres como mujeres, aunque por las características sociodemográficas de la profesión, se anticipaba que serían más maestras que maestros. Sin embargo, no se encontró ningún hombre que cumpliera las características de trabajar como maestro y ser un practicante sistemático de yoga. Entre los profesores se contó con la participación de tres maestros (varones) y de dos maestras de yoga profesionales, con una mayor experiencia en esta práctica meditativa, que, eventualmente, impartían clases de esta disciplina, bien a adultos, bien a niños o a ambos. Se pretendía con ello posibilitar la triangulación de datos desde participantes distintos. 
La técnica de recogida de datos que se entendió adecuada fue la entrevista semiestructurada y en profundidad. A través de la entrevista, la comunicación pone en juego las visiones de los sujetos implicados, en este caso, de investigador y de participante investigado. En la entrevista interesan los diversos puntos de vista, así como tener en cuenta tanto las perspectivas "emic" y "etic" (Harris, 1980), es decir la descripción subjetiva de los participantes ("emic") y del discurso de la teoría o de los expertos ("etic"). Afirma Bourdieu (2006) que, en el marco de la entrevista, sólo la reflexividad, comprendida como método, presenta las condiciones para la objetivación científica del sujeto. El hecho de narrar sobre uno mismo y las propias vivencias, obliga a tomar conciencia de sí, de los sentimientos, pensamientos, creencias, sentido, etc., ayudando reactivamente a la construcción y toma de conciencia de nuevos conocimientos y saberes. Desde la formación actual, se narra el pasado, se resignifica el presente y se sistematizan y comunican conocimientos (significados) que lo describen, cuestionan y explican para la mejora personal y profesional.

Para Bourdieu (2006), el investigador debe esbozar estrategias para ayudar al entrevistado a descubrir su verdad. Según Contreras y Pérez de Lara (2010) el valor de verdad de una investigación descansa en:

La autenticidad de la relación que se establece entre las reflexiones pedagógicas y la forma en que éstas se han generado en el diálogo con la realidad estudiada; en la honestidad de afrontar la propia subjetividad mientras se busca conocer y dejarse afectar por los acontecimientos; en mostrar la multiplicidad de significados posibles y la forma de generarse preguntas, enseñando la manera en que nacen y se relacionan con lo vivido (p. 71)

El análisis de los datos en una investigación cualitativa de este tipo pretende conocer el saber experimental y subjetivo, que nace de todas las facetas humanas particulares y dialógicas. El procedimiento para su realización fue un análisis de contenido, donde se busca el significado, apoyado en razones, expresión y testimonios de los casos participantes. Según Bardin (1996), el análisis de contenido sería:

el conjunto de técnicas de análisis de las comunicaciones tendentes a obtener indicadores (cuantitativos o no) por procedimientos sistemáticos y objetivos de descripción del contenido de los mensajes permitiendo la inferencia de conocimientos relativos a las condiciones de producción/ recepción (contexto social) de estos mensajes (p. 32) 
Las entrevistas fueron grabadas y posteriormente transcritas, previa autorización y consentimiento informado de todos los entrevistados. El análisis de contenido se realizó con el apoyo del programa Atlas.ti. Las respuestas se codificaron, categorizaron y subcategorizaron inductiva y deductivamente (Fereday, 2006).

\section{Resultados y discusión}

El sistema de categorías en esta investigación fue mixto, es decir, se estableció de forma emergente y sobre la base de categorías definidas a priori. Las categorías fueron redefinidas, reorganizadas, comparadas y relacionadas en una red de significados, según los testimonios de los participantes (Figura 2).

Se reflejarán a continuación solo los resultados unánimes o coincidentes en, al menos, tres cuartas partes de los participantes.

No se apreció ninguna diferencia, ni patrones de respuesta diferenciales entre los profesores de infantil y primaria practicantes sistemáticos de yoga y los que, además, eran profesores de yoga con adultos o con niños.

La mayor o menor práctica requerida para la selección de los participantes no influyó decisivamente en sus expresiones o en el contenido de sus argumentaciones.

Las definiciones que los maestros de infantil y primaria dan de 'conciencia' se acercan bastante a las de los profesionales del yoga y a las reunidas en el marco teórico: "La conciencia es lo que realmente somos" (P. 6). "Para mí, la conciencia es esa actitud de permanecer alerta para discernir el camino de la sabiduría" (P. 5). "La atención y la percepción vivas y centradas en lo que se está haciendo" (P. 8).

Así, desde un punto de vista "etic", la conciencia se interpreta como autoconciencia, conciencia del otro y conciencia de sistemas más amplios (Goleman y Senge, 2016). Sin embargo, la categoría semántica más recurrente es la de autoconciencia. No obstante, los participantes relacionan la autoconciencia con la conciencia del otro. Todos los participantes coinciden con Goleman y Senge (2016), en que la primera en la base de la segunda. Las definiciones referidas a la conciencia de sistemas más amplios se dan en mucha menor proporción. 


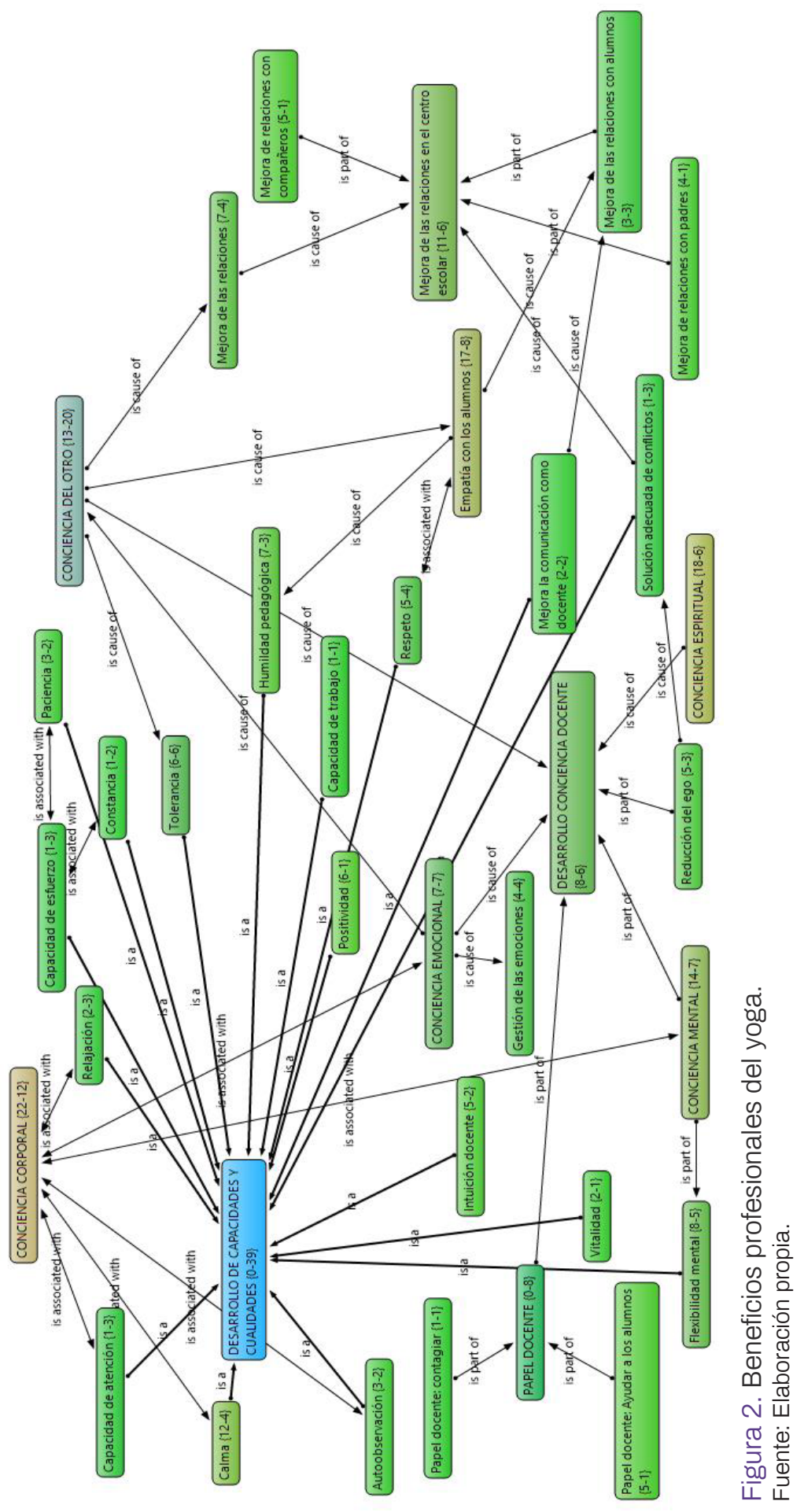


Los participantes consideran la conciencia como una entidad más allá de la mente, como la actividad cerebral de darse cuenta, proceso de presencia y conexión, facultad humana: comprensión, autoconocimiento y sabiduría y la relacionan con cualidades humanas como la responsabilidad y la coherencia. "Desde la conexión con el mundo y lo superior, hasta la responsabilidad de cada uno, lo que conlleva a que cada cosa que yo hago tenga una consecuencia" (P. 1). Coinciden, desde este conocimiento experimental, con Calle (2017) y la teoría del yoga, que conceptúan la conciencia profunda más allá de la mente.

Todos estos maestros consideran que la conciencia es un factor muy importante en la formación y en el desarrollo profesional y personal del docente. Este consenso coincide con el enfoque radical e inclusivo, que afirman que la formación es en términos de más y más conciencia aplicada al ámbito o contenido de que se trata, y que el desarrollo personal y personal o formativo lo es en la medida en que se produce una evolución del egocentrismo a la conciencia (Herrán, 1995, 1997, 1998, 2011b). En cuanto a esta relación concreta de la conciencia con la docencia, se aíslan dos dimensiones de análisis, surgidas de los datos: Conciencia docente y conciencia educativa.

La conciencia docente implica un papel menos directivo y asocia el desarrollo de cualidades del educador, como la responsabilidad, la coherencia, la madurez docente, la empatía, la humildad didáctica, el amor por los alumnos y la confianza en los demás y en sí mismo. Para Day (2006), la profesión docente tiene que ver sobre todo con el amor. Coincide con la mirada filosófica profunda de Lledó (1999). El afecto y la compasión son, para Day (2006), características esenciales del establecimiento y mantenimiento de la conexión con los alumnos y los compañeros de trabajo. Desde un punto de vista didáctico, precisa que: "El comportamiento afectivo guía la atención del alumno, lo que es determinante del rendimiento, por lo que es probable que las relaciones emocionales positivas reduzcan la incidencia de problemas conductuales y aumenten la motivación por aprender." (Day, 2006, p. 67)

La conciencia pedagógica, entendida como la que los docentes tienen sobre la educación, se concreta en su modo de entender la finalidad educativa. En los profesores practicantes de yoga que participaron en el estudio, el sentido de la educación tiene que ver, sobre todo, con desarrollar la conciencia $y$, desde ella, cultivar las cualidades humanas e innatas del niño, como la libertad, la bondad o la felicidad. En un segundo plano, pero sin relegarlas, 
quedan cuestiones más asimilables a la instrucción, como el aprendizaje intelectual de contenidos o el desarrollo de competencias. Se considera importante el desarrollo de la capacidad intelectual, la atención y la flexibilidad mental, un factor de la creatividad. En este sentido, coinciden con la tesis de Krishnamurti (2016) en que la libertad está en el hecho de aprender a observar con atención el mundo que le rodea:

Lo importante en educación es despertar en el alumno la inteligencia, el amor, la compasión y la libertad interior. La libertad, no como el acto de decidir, sino en el acto de percibir sin condicionamientos del pasado, de nuestro sistema de creencias y de juicios (Krishnamurti, 2016).

Otras capacidades que los docentes dicen necesario desarrollar en los niños son la coherencia, que surge del equilibrio dinámico entre los diferentes tipos de capacidades: amor, bondad, sensibilidad, relacionadas con la capacidad de empatía, y la intuición y flexibilidad mental, relacionadas con la sabiduría que el niño tiene desde su infancia, coincidiendo en esto con Herrán (2015). En síntesis, para los participantes lo más importante a desarrollar en el niño es el cultivo de todas las capacidades humanas de forma integral, armónica y equilibrada: sociales, afectivas-emocionales, cognitivas o intelectuales, corporales y morales-espirituales. Teniendo en cuenta que se trata de sacar de dentro del niño la sabiduría que tiene, englobaría aspectos de todas ellas cuando surgieran de la conexión consigo mismo, mediante la atención aplicada a la práctica del yoga y mediante el desarrollo de la conciencia.

Coinciden con Ederra (2017) en que así la sabiduría del niño estaría íntimamente ligada al autoconocimiento y a la intuición:

La intuición es un puente a otra sabiduría que, lejos de contribuir a la separatividad, interrelaciona y unifica al ser humano. La intuición ilumina nuestra mente analítica y ensancha nuestro corazón. Se cultiva a través del silencio interno de la mente, y potencia el amor, la fuerza de voluntad y la conciencia. Como docentes, puede guiarnos y transmitirnos aquello que necesitamos crear, desarrollar o aplicar en el aula a cada momento, para ofrecer experiencias de aprendizaje verdaderamente significativas al alumno, en las que su propia sabiduría interna sea estimulada, en las que aprenda por descubrimiento propio y desarrolle sus cualidades e inteligencias múltiples a través de su propia intuición, creatividad y conciencia. (p. 16). 
Más allá del plano de sus alumnos, pero dentro de la cuestión del fin de la educación, para la totalidad de los docentes, el sentido último de la educación se correspondería con la evolución del ser humano o de la humanidad. No hay respuestas que se detengan en el desarrollo de un sistema menor (nacional, geográfico, religioso, educativo, etc.).

En cuanto a los beneficios que los profesores practicantes de yoga consideran que esta disciplina les ha aportado, tanto en los planos personal como docente, la mayoría de ellos respondieron más allá de la dualidad planteada. Los testimonios mayoritarios apuntaron a un beneficio directo en el plano personal que, no obstante, se manifestaba en el trabajo. Estos testimonios sintéticos confirman la tesis de que el desarrollo profesional del docente requiere una previa madurez de la persona Fernández (2007) o de que no hay desarrollo profesional sin desarrollo personal en la docencia (Herrán, 2008).

Desde la perspectiva de los profesores practicantes, la clase de conciencia que el yoga ayuda a desarrollar con más fuerza es la conciencia de sí, especialmente la emocional y corporal. Sin embargo, la mitad de los participantes se refiere a la conciencia espiritual, que interpretan como experiencia de conexión con el mundo y lo superior, que conduce al autoconocimiento verdadero mediante la conciencia.

En ninguno de los casos hay equivalencia entre lo superior y Dios, la Divinidad o similares; más bien se relaciona con la naturaleza, el universo, la vida. Esta conciencia espiritual se correspondería con la conciencia transpersonal de la que hablan los autores de la teoría transpersonal (Wilber, 2007), que equivaldría al más alto nivel de desarrollo de la conciencia. La conciencia espiritual se identifica con la moral autónoma (Kohlberg, Clark y Higgins, 1997), cuyos indicadores tienen que ver con el darse cuenta de los propios valores, de lo que está bien o mal de forma autónoma, sin reglas impuestas desde fuera. En un sentido compatible, Osho (2010) afirma que cuando se es consciente la bondad se convierte en el modo habitual de ser, y el bien viene por sí solo, para lo cual solo hay que ir hacia adentro. En este sentido, el autor critica la "conciencia moral", que conceptúa como una estrategia de la sociedad para esclavizar a la persona (Osho, 2010, p. 180). 
Todos los participantes expresaron que la práctica sistemática del yoga les ayudaba directa y concretamente al desarrollo de la conciencia del otro. Destacaron que el incremento de la conciencia del otro era esencial en el trabajo docente, por ser una tarea esencialmente comunicativa. Se extendió la tarea comunicativa tanto con los miembros del equipo educativo, familias y alumnos. Unánimemente consideran que el yoga les había ayudado a mejorar, al volverse más empáticos y, por tanto, más conscientes de sus necesidades: "Miro con más distancia los roces personales y el ego entre los profesores y tiendo a ser más indulgente o menos estricta con los alumnos" (P. 2). "A nivel personal, me ha ayudado mucho a escuchar a los niños, a percibir su potencial y a restarme yo en importancia, humildemente." (P. 3)

También hubo acuerdo en que el yoga fue útil para incrementar cualidades pedagógicas de los docentes. Algunas cualidades a las que el yoga ayudó a desarrollar, según los maestros, fueron las que consideraban esenciales para la labor docente: amor, atención, coherencia, empatía, humildad, responsabilidad (constancia, capacidad de trabajo y esfuerzo), confianza y presencia. Esto confirma la valoración del amor de Day (2006) o de Lledó (1999), entre otras. La percepción de ganancia de atención y de empatía coincide con Walsh y Vaughan (2003), que recogen: "investigaciones que sugieren que los procesos perceptuales de los meditadores son más sensibles y más rápidos y que su empatía se agudiza" (p. 96). De la empatía surge la también citada por varios participantes humildad (didáctica), que incrementa la posibilidad de atención y apertura a personas (alumnos y otros educadores: profesores y padres) y a situaciones, y a aprender de ellas. Así mismo, los maestros practicantes de yoga observaron que había otras cualidades relacionadas con la empatía que la práctica del yoga había potenciado en su enseñanza del día a día: tolerancia y capacidad de comunicación y relación.

También expresaron haber sido fortalecidas por la práctica sistemática del yoga la paciencia, la fuerza interna, la aceptación y la positividad, interpretable como energía y optimismo. Todas ellas son cualidades relacionadas con la resiliencia que, según Aguaded y Almeida (2016), son rasgos importantes para el docente, en cuyo trabajo pueden experimentarse episodios de frustración o de dureza. Los testimonios apuntaron a un incremento de la capacidad de disfrute, por ganancia de sensibilidad, dependiente de la capacidad de sentir bienestar con independencia de estímulos exteriores. Este 
efecto es relevante, porque, si se es capaz de disfrutar de lo que estamos haciendo, será más fácil transmitirlo a alumnos y familias, y se incrementará la posibilidad de desarrollo personal y profesional.

En cuanto a los cambios que provoca el yoga en la conciencia aplicada a la educación, se concretaron en una interpretación diferente de la finalidad educativa, traducible en la importancia de trabajar en el desarrollo de la conciencia del niño, la conciencia sobre los elementos pedagógicos y la preocupación por la introducción de elementos del yoga en la clase, para el beneficio del alumno, de la formación y de la instrucción. Se alude a la falta de conciencia educativa aplicada, tanto a la educación como en las leyes, en currículo escolar y en la formación de los profesores, coincidiendo con Herrán (1998). La justificación u observación principal se refiere, precisamente, a que no parece haber noción clara de lo importante que es desarrollar la conciencia para cada ser humano en particular y para la sociedad global.

De acuerdo con la finalidad educativa a la que unánimemente apuntan los maestros -el desarrollo de la conciencia de los alumnos-, la enseñanza debería consistir en sacar lo que el niño ya tiene, como potencial condensado en una sabiduría innata. Los maestros resaltan la importancia de tener en cuenta las necesidades de los niños y su motivación, de crear metodologías vivas, con base en la conciencia, por medio del silencio y la práctica de la atención consciente. Afirman que el yoga les ha permitido ser conscientes de que los libros de texto, las metodologías y los exámenes no respetan al niño. El respeto, que señalaban casi todos los docentes como elemento importante en la educación del niño, no se está teniendo en cuenta con determinados elementos pedagógicos, que en muchas ocasiones vienen impuestos desde fuera. Esta percepción confirma la relevancia del "respeto didáctico" (Herrán y González, 2002) como factor de una enseñanza formativa.

Finalmente, los practicantes coincidieron en que el yoga enseña, sobre todo, que todo está unido en nuestro ser, y que son circunstancias externas las que nos separan de nosotros mismos, el resto de los seres y la naturaleza. De ahí que el yoga contribuya a ampliar, profundizar, equilibrar, afinar, serenar y relacionar lo próximo con la conciencia ecológica. Los profesores practicantes de yoga comparten una conciencia sociopolítica que traspasa las fronteras del aula y de los sistemas egocéntricos, y una relación con la evolución social y de la humanidad. Desde esta percepción amplia y no parcial, se observan los problemas concretos, tanto sociales como medioambientales. 


\section{Conclusiones}

A partir del objetivo principal del estudio y del análisis de los resultados se deducen algunas conclusiones preliminares.

La primera, de carácter gnoseológico, confirma que las descripciones y testimonios "emic" (subjetivas y experimentales) de los participantes verifican conclusiones y tesis "etic" (teóricas), desde las que cabría observar una validación de su contenido, en sentido estricto.

En segundo lugar, el yoga es una metodología formativa eficaz, desde la perspectiva de los participantes. En efecto, para los profesores practicantes de yoga, esta disciplina meditativa ayuda a fomentar cualidades formativas relevantes de los docentes, ratificadas por teóricos e investigadores de la educación. Todas ellas se consideran necesarias para el desarrollo personal y profesional del docente y esenciales para el trabajo docente. Los más relevantes son: positividad (energía y optimismo), capacidad de esfuerzo, fortaleza, capacidad de relacionarse y amplitud y flexibilidad mental.

En tercer lugar, la práctica del yoga desarrolla la conciencia del docente, entendida tanto como autoconciencia, como conciencia del otro, aspecto esencial en la profesión docente, y, en menor medida, la conciencia de sistemas más amplios.

Por otra parte, el yoga ayuda a ver claramente que el fin de la educación y la enseñanza pasa por el desarrollo de la conciencia, comprendida como el factor más importante de la educación, junto al control del egocentrismo humano (Herrán, 1995, 2014). Se traduce en el desarrollo integral de las capacidades, la priorización de aspectos humanos y emocionales sobre los contenidos escolares, la importancia de cambiar el modo de entender el aprendizaje, que no es extrínseco, sino relacionado con sacar lo que el niño ya lleva dentro, aunque se apoye en libros de texto, otros recursos, metodologías directivas, exámenes, etc. Los participantes comparten la convicción de que la educación es la base de las soluciones, pero por educación no entienden la intervención que fomenta la adaptación personal a los sistemas políticos y económicos, perpetuando desigualdades y contribuyendo al deterioro de nuestro medio ambiente. Más bien identifican educación como proceso de adquisición y desarrollo de conciencia. 
En quinto lugar, los resultados apuntan a que la práctica del yoga contribuye al desarrollo personal y profesional del docente, desde el fortalecimiento de su conciencia e, indirectamente, a la educación del alumno. En efecto, al variar la perspectiva con la que el docente mira y ve la educación y a los alumnos, se mejora espontánea, automáticamente la relación con ellos, con las familias y con los compañeros de trabajo. En consecuencia, se considera positiva y conveniente la práctica del yoga, comprendida como una disciplina o vía de desarrollo de la conciencia.

Globalmente, los resultados confirman la tesis del enfoque radical e inclusivo de la formación que apunta a que, para la mejora de la enseñanza, centrarse en la práctica y en la reflexión sobre la práctica podría ser un error de planteamiento, por ser, ambos, efectos de quien realiza esa práctica. La alternativa formativa complementaria es centrarse en la causa o fuente de la reflexión o de la práctica, esto es, en el ego docente y la conciencia de quien reflexiona y desarrolla la práctica e, idealmente, en su cultivo formativo meditativo. Los resultados coinciden con el enfoque radical e inclusivo de la formación en cuatro necesidades y cambios epistemológicos: (1) Redefinir y complementar los fines de la educación, tal y como normalmente se entienden, desde la Pedagogía (Monarca, 2009). (2) Atender e incluir enfoques formativos complementarios con base en la conciencia, junto a los modos en que habitualmente se forma a los profesores, desde una perspectiva técnico-reflexiva. (3) Incluir constructos educativos claves, como el ego docente (Herrán y González, 2002), la conciencia (Herrán, 1998), el autoconocimiento esencial (Herrán, 2004), los condicionamientos mentales (Krishnamurti, 2016), etc. (4) Favorecer el tránsito en complejidad de una educación y una enseñanza basadas en el aprendizaje a una educación y una didáctica basadas en la conciencia, sin renunciar al aprendizaje. Desde el enfoque radical e inclusivo de la formación, la pregunta clave no es si el yoga pudiera o no incluirse en la formación del profesorado, sino ¿cómo es posible que una metodología autoformativa tan contrastada todavía permanezca tan lejos de la educación en general y de la formación del educador y del profesorado, en particular, así como de las ciencias que los estudian, con especial referencia a la Pedagogía y la Didáctica (Herrán, 1995)?

Los resultados apuntan a que la práctica sistemática del yoga puede ser una metodología importante para el profesorado y la educación. Potencia, además, numerosas cualidades de los profesores. En especial, redunda en 
una mejor relación interpersonal y educación de los alumnos. Esta forma de meditación es altamente compatible con el enfoque técnico reflexivo. El yoga se muestra, así, como una práctica del máximo interés pedagógico.

\section{Referencias}

Aguaded, M. C. y Almeida, N. A. (2016). La resiliencia del docente como factor crucial para superar las adversidades en una sociedad de cambios. Tendencias Pedagógicas 28, 167-180. https://doi.org/10.15366/tp2016.28.012

Aguayo, J. (2013). Investigación en la escuela: el sentido de los datos desde la tradición comprensiva. México: UNAM.

Bardin, L. ( 1996). Análisis de contenido. (2ed). Barcelona: Akal.

Bourdieu, P. (2006). Autoanálisis de un sociólogo. Barcelona: Anagrama.

Buber, M. (1977). Yo y tú. Buenos Aires: Nueva Visión.

Buda (1997). Dhammapada o las enseñanzas de Buda. México: FCE.

Calle, R. (2017) Los 7 yogas. Barcelona: Integral.

Confucio (1969). Los cuatro libros de Confucio. Madrid: Bergua.

Contreras, J. \& Pérez de Lara, N. (2010) La experiencia y la investigación educativa. En J. Contreras y N. Pérez de Lara (Comps.) (pp. 21-86). Investigar la experiencia educativa. Madrid: Morata.

Correa, M. L. y Guerra, S. (2014). El bienestar psicológico en participantes de yoga, biodanza y sudarshan kriya yoga. Tesis de Licenciatura. Facultad de Psicología. Pontificia Universidad Javeriana, Bogotá (Colombia).

Damasio, A. (2010). Antonio Damasio: el mago del cerebro. El país digital (10/11/2010).

Day, C. (2006). La pasión por enseñar: La identidad personal y profesional del docente y sus valores. Madrid: Narcea.

Deniz, J. L., Torres, M. L.\& Alcantar, R. (2010). La salud físico-emocional de los profesores de la Universidad de Colima. Recuperado de https://bit.ly/2ObQKvD

Ederra, M. (2017). Meditación e intuición. En A. de la Herrán (Coord.). Meditación y educación. Boletín del Ilustre Colegio Oficial de Doctores y Licenciados en Filosofía y Letras y en Ciencias de Madrid. Apuntes de Pedagogía (269), 14-16. Recuperado de https:// bit.ly/3kBZCqy

Fereday, J. (2006). Demonstrating rigor using thematic analysis: A hybrid approach of inductive and deductive coding and theme development. International Journal of Qualitative Methods, 5(1), 80-92.

Fernández, A. (2007). El desarrollo profesional del docente y el perfil del docente. El Salvador: Universidad Centroamericana "José Simeón Cañas".

Fromm, E. (1991). Del tener al ser. Caminos y extravíos de la conciencia. Barcelona: Paidós. 
Gimeno, J. (1998). Poderes inestables en educación. Madrid: Morata.

Goleman, D. y Senge, P. (2016). Triple Focus. Barcelona: Editorial B. (e. o. 2014)

González, F. E. (2008). Qué es y de qué se ocupa la Didáctica: Sus fundamentos y métodos. En A. de la Herrán, y J. Paredes (Coords.), Didáctica General. La práctica de la enseñanza en educación infantil, primaria y secundaria (pp. 1-26). Madrid: Mc Graw-Hill Interamericana.

Gónzalez-Zarza, A. M. (2006). El desarrollo de la conciencia desde la óptica transpersonal. En Almendro, M. (ed.). La conciencia transpersonal $\left(2^{a}\right)$ (pp.89-98) Barcelona: Kairós.

Harris, M. (1980). Cultural materialism: the struggle for a science of culture. New York: Random House.

Herrán, A. de la (1993). La educación del siglo XXI. Cambio y evolución humana. Madrid: Ciencia 3.

Herrán, A. de la (1995). Ego, autoconocimiento y conciencia: Tres ámbitos en la formación básica y la evolución personal de los profesores. Madrid: Universidad Complutense de Madrid. Servicio de Publicaciones.

Herrán, A. de la (1996). Hacia otra nueva educación. Boletín del llustre Colegio Oficial de Doctores y Licenciados en Filosofía y Letras y en Ciencias de Madrid (75), 24-25.

Herrán, A. de la (1997). El ego humano. Madrid: San Pablo.

Herrán, A. de la (1998). La conciencia humana. Madrid: San Pablo.

Herrán, A. de la (2003). El siglo de la educación. Formación evolucionista para el cambio social. Huelva: Hergué.

Herrán, A. de la (2004). El autoconocimiento como eje de la formación. Revista Complutense de Educación, 15(1), 11-50. Recuperado de https://bit.ly/3dVnxj8

Herrán, A. de la (2008). El profesor que se forma: desarrollo personal y profesional del docente. En J. C. Sánchez Huete, Compendio de Didáctica General (pp. 109-152). Madrid: CCS.

Herrán, A. de la (2011a). ¿Más allá del profesor reflexivo y de la reflexión sobre la práctica? En A. Medina, A. de la Herrán y C. Sánchez (Coords.), Formación pedagógica y práctica del profesorado (pp. 117-152). Madrid: Ramón Areces.

Herrán, A. de la (2011b). Reflexiones para una reforma profunda de la educación, desde un enfoque basado en la complejidad, la universalidad y la conciencia. Educación XX1, 14, 245-264. Recuperado de https://bit.ly/3bRnnXI

Herrán, A. de la (2014). Enfoque radical e inclusivo de la formación. REICE. Revista Iberoamericana sobre calidad y cambio en educación, 12(2), 163-264. Recuperado de https://bit. ly/3sApW74

Herrán, A. de la (2015). ¿El niño como maestro? En J. L. Villena Higueras y E. Molina Fernández, Ciudades con vida: Infancia, participación y movilidad (pp. 103-108). Barcelona: Graó. Recuperado de https://bit.ly/3b3NZ8m

Herrán, A. de la (2016a). La mala práctica educativa desde el enfoque radical de la formación. Boletín del Ilustre Colegio Oficial de Doctores y Licenciados en Filosofía y Letras y en Ciencias de Madrid (267), 10-12. Recuperado de https://bit.ly/2NFFEPD

Herrán, A. de la (2016b). Reflexiones pedagógicas desde el enfoque radical e inclusivo de la formación. Salamanca: Fahrenhouse. Recuperado de https://bit.ly/3uBXQKB 
Herrán, A. de la (2017a). ¿Y si nuestra educación estuviese radicalmente equivocada? En A. Medina, A. de la Herrán Gascón y M. C. Domínguez (Coords.), Nuevas perspectivas en la formación de profesores. Madrid: UNED. Recuperado de https://bit.ly/3bMRhw0

Herrán, A. de la (2017b). Para una Pedagogía radical e inclusiva. Editorial Revista Virtual Redipe (7), 24-32. Recuperado de https://bit.ly/3uHG20B

Herrán, A. de la (2017c). Pedagogía radical e inclusiva. Primera parte: Problemas de la educación y la formación. En M. C. Domínguez Garrido, R. Ferreiro Gravié, A. de la Herrán Gascón, M. Medina Domínguez y A. Medina Rivilla. Hacia una Didáctica del siglo XXI. Zapopan (Jalisco): Universidad Santander (UNISAN). Recuperado de https:// bit.ly/3b2laHm

Herrán, A. de la (2017d). Pedagogía radical e inclusiva. Segunda parte: Alternativas para la educación y la formación. En M. C. Domínguez Garrido, R. Ferreiro Gravié, A. de la Herrán Gascón, M. Medina Domínguez y A. Medina Rivilla. Hacia una Didáctica del siglo XXI. Zapopan (Jalisco): Universidad Santander (UNISAN). Recuperado de https://bit.ly/37XZwnU

Herrán, A. de la (2017e). Sin meditación no hay educación. Boletín del Ilustre Colegio Oficial de Doctores y Licenciados en Filosofía y Letras y el Ciencias de Madrid. Apuntes de Pedagogía (269), 24-26. Recuperado de https://bit.ly/3uK53ID

Herrán, A. de la (2018). Fundamentos para una Pedagogía del saber y del no saber. São Paulo: Edições Hipótese. Recuperado de http://bit.ly/3sBWloj

Herrán, A. de la y González, I. (2002). El ego docente, punto ciego de la enseñanza, el desarrollo profesional y la formación del profesorado. Madrid: Universitas.

Iyengar, B. K. (2007) Luz sobre el yoga. Yoga Dipika. Barcelona: Kairós.

Jyoti, J., Rani, R. \& Gandotra, R. (2015) The impact of bundled high performance human resource practices on intention to leave: Mediating role of emotional exhaustion. International Journal of Educational Management, 29(4), 431-460.

Kohlberg, L., Clark, F. \& Higgins, A. (1997) La educación moral según Lawrence Kohlberg . Barcelona: Gedisa.

Kornfield J. (2003) Los siete factores de la iluminación. En R. Walsh y F. Vaughan (Comp.) Trascender el ego: La visión transpersonal. (2ª ed.) (pp. 105-111) Barcelona: Kairós.

Korthagen, A. J. (2010). La práctica, la teoría y la persona en la formación del profesorado. Revista Interuniversitaria de Formación del Profesorado, 68(24,2), 83-101. Recuperado de http://bit.ly/2skdNt9

Krishnamurti, J. ( 2016). La revolución interior. Barcelona: Kairós.

Lledó, E. (1999). Lledó: "La única Pedagogía que existe es el amor". Revista Escuela Española, 3408.

Martínez, J. (1988). El estudio de caso en la investigación educativa. Revista Investigación en la Escuela, 6, 41-50.

Monarca, H. (2009). Los fines de la educación. Sobre la necesidad de recuperar y revisar el debate teleológico. Madrid: Narcea.

Osho. (2010). Cierra los ojos y lánzate (1ª ed.). Barcelona: Debolsillo. 
Pérez, A. I. (2010). Nuevas exigencias y escenarios para la profesión docente en la era de la era de la información y de la incertidumbre. Revista interuniversitaria de formación del profesorado, 24(2), 17-36. Recuperado de http://bit.ly/2Pe6MFC

Platón (1969). Apología de Sókrates. Madrid: Bergua.

Ramírez Vallejo, M. S. y Herrán, A. de la (2012). La madurez personal en el desarrollo profesional del docente. REICE. Revista Iberoamericana sobre Calidad, Eficacia y Cambio en Educación, 10(3), 25-44. Recuperado de https://bit.ly/2ZZ2807

Sanches, A. (2008). Educar es creer en la persona. Madrid: Narcea.

Tójar, J. (2006) Investigación cualitativa. Comprender y actuar. Madrid: Muralla.

Torre, S. de la (2010) Adversidad y diversidad creadoras. Desarrollando otra conciencia. En S. de la Torre y M. A.Pujol (Comps.) Creatividad e innovación. Enseñar e investigar con otra conciencia (pp. 19-34). Madrid: Universitas.

Walsh, R. y Vaughan, F. (2003). Trascender elego: la visión transpersonal (2ª ed). Barcelona: Kairós.

Wilber, K. (2007) Espiritualidad integral el nuevo papel de la religión en el mundo actual. Barcelona: Kairós.

Yin, R. (2009) Case study research. design and methods (4ª ed.). London: Sage. 